\title{
On coherent-state representations of quantum mechanics: Wave mechanics in phase space
}

Møller, Klaus Braagaard; Jørgensen, Thomas Godsk; Torres-Vega, Gabino

Published in:

Journal of Chemical Physics

Link to article, DOI:

10.1063/1.473684

Publication date:

1997

Document Version

Publisher's PDF, also known as Version of record

Link back to DTU Orbit

Citation (APA):

Møller, K. B., Jørgensen, T. G., \& Torres-Vega, G. (1997). On coherent-state representations of quantum mechanics: Wave mechanics in phase space. Journal of Chemical Physics, 106(17), 7228-7240.

https://doi.org/10.1063/1.473684

\section{General rights}

Copyright and moral rights for the publications made accessible in the public portal are retained by the authors and/or other copyright owners and it is a condition of accessing publications that users recognise and abide by the legal requirements associated with these rights.

- Users may download and print one copy of any publication from the public portal for the purpose of private study or research.

- You may not further distribute the material or use it for any profit-making activity or commercial gain

- You may freely distribute the URL identifying the publication in the public portal 


\title{
On coherent-state representations of quantum mechanics: Wave mechanics in phase space
}

\author{
Klaus B. Møller and Thomas G. Jørgensen \\ Department of Chemistry, Chemical Physics, Technical University of Denmark, DTU-207, \\ DK-2800 Lyngby, Denmark \\ Gabino Torres-Vega \\ Departamento de Física, Centro de Investigación y de Estudios Avanzados del IPN, Apartado Postal \\ 14-740, 07000 México, D.F. Mexico
}

(Received 2 January 1997; accepted 27 January 1997)

\begin{abstract}
In this article we argue that the state-vector phase-space representation recently proposed by Torres-Vega and co-workers [introduced in J. Chem. Phys. 98, 3103 (1993)] coincides with the totality of coherent-state representations for the Heisenberg-Weyl group. This fact leads to ambiguities when one wants to solve the stationary Schrödinger equation in phase space and we devise two schemes for the removal of these ambiguities. The physical interpretation of the phase-space wave functions is discussed and a procedure for computing expectation values as integrals over phase space is presented. Our formal points are illustrated by two examples. (C) 1997 American Institute of Physics. [S0021-9606(97)02317-9]
\end{abstract}

\section{INTRODUCTION}

Phase space is a fundamental concept in Hamiltonian mechanics and phase-space formulations of quantum mechanics are therefore of great interest in order to compare quantum and Hamiltonian mechanics, for finite $\hbar$ and when $\hbar$ goes to zero. However, due to the uncertainty principle caution is required when one wants to consider position and momentum together in quantum mechanics.

The usual Schrödinger representation of quantum mechanics diagonalizes the position operator, i.e., it is multiplicative, a quantum state is represented as a wave function that depends on the position variable, with the momentum operator being a differential operator, that is, it is non-local. This representation can be Fourier transformed into a momentum representation that diagonalizes the momentum operator, a quantum state is represented as a wave function that depends on the momentum variable, and the position operator becomes non-local.

Many attempts to a phase-space description of quantum mechanics have been made, the most famous ones being due to Fock $^{1}$ (his representation has been studied thoroughly by Bargmann $^{2}$ ), Wigner ${ }^{3}$ and Husimi. ${ }^{4}$ Recent reviews on the Fock-Bargmann representation can be found in Refs. 5 and 6 and for recent reviews on the Wigner and Husimi representations see e.g., Refs. 7 and 8. Moreover, Harriman and Casida $^{8}$ analyze the Wigner and Husimi distributions in the limit of a small $\hbar$ (see also e.g., Refs. 9 and 10). A semiclassical study using the Fock-Bargmann representation is given, for instance, by Voros. ${ }^{11}$

The Fock-Bargmann representation is a state-vector representation that diagonalizes the non-Hermitian creation operator, where a quantum state is represented as an entire (wave) function of a complex variable, and where the annihilation operator is non-local. In this representation the equations of motion are of the Schrödinger type. This should be contrasted to the phase-space representations of Wigner and
Husimi where a quantum state is represented as a distribution function, observables are represented by functions, and the equations of motion are of the Liouville type. These representations, of course, all have a right on their own in the sense that a quantum problem can be solved entirely within the representation, but they can also be obtained from the Schrödinger representation, in which case the FockBargmann wave function is obtained from the Schrödinger wave function by a linear map whereas the Wigner and $\mathrm{Hu}-$ simi distributions are bilinear in the Schödinger wave function. However, the Fock-Bargmann and the Husimi representations are closely related in that the Husimi distribution equals the square magnitude of the corresponding FockBargmann wave function times $\exp \left(-|z|^{2}\right)$.

Recently, there has been renewed interest in a quantum state-vector phase-space representation (SVPSR). ${ }^{12-17}$ Unlike the Fock-Bargmann representation, this SVPSR is formulated in terms of the real "phase-space" coordinates $q$ and $p$ and operator mappings are given for the fundamental operators $Q$ and $P$. In Ref. 12 Torres-Vega and Frederick postulate the existence of a complete set of vectors $|q, p\rangle$ that may serve as a basis such that a quantum state $|\psi\rangle$ can be represented in phase space by an $\mathscr{D}^{2}(2)$ wave function $\psi(q, p)=\langle q, p \mid \psi\rangle$ and the operators $Q$ and $P$ in this basis take the (non-local) forms

$$
\begin{aligned}
& Q \mapsto\left(\frac{q}{2}+i \hbar \frac{\partial}{\partial p}\right), \\
& P \mapsto\left(\frac{p}{2}-i \hbar \frac{\partial}{\partial q}\right) .
\end{aligned}
$$

With this representation it appears that Torres-Vega and Frederick have obtained a wave-mechanical formulation of quantum mechanics in phase space similar to the usual ones in position and momentum space; for the phase-space wave function the equation of motion is of the Schrödinger type, 
and its square magnitude may play the role of a density in phase space whose dynamics is controlled by a Liouville type equation. However, the SVPSR based on the operator mapping Eq. (1) differs from representations in position and momentum space on one important point - it is not unique. The same quantum state is represented by an infinite number of different wave functions in phase space. This may lead to some difficulties. For instance, it raises the question about how to interpret the square magnitude of a phase-space wave function as a density.

Previously Torres-Vega and co-workers have found examples of phase-space wave functions representing stationary states for linear and quadratic potentials and analyzed them for certain features; for instance, whether they are stationary solutions to the classical Liouville equation. ${ }^{16,17}$

The purpose of the present work is to discuss in more detail the origin of the operator mapping in Eq. (1) and analyze the implications of our findings on the interpretation of the square magnitude of a phase-space wave function as a density and the possibilities of doing wave mechanics in phase space using Eq. (1). The reason for the ambiguity in a phase-space representation based on Eq. (1) is that there exists an infinite number of complete bases parametrized by $q$ and $p$ that give rise the operator mapping in Eq. (1). In Diracian sense this implies that we are dealing with an infinite number of different representations of quantum mechanics that are, in principle, independent. However, since they all give the same operator mapping the bases defining each representation must be somehow similar. In fact, this similarity is well-known. Any set of coherent states, as formulated by Klauder and Skagerstam, ${ }^{5}$ used as a basis in a SVPSR will result in the operator mapping in Eq. (1). ${ }^{5}$ The perhaps most familiar set of coherent states is the Glauber coherent states. ${ }^{18}$ The use of this set as a basis in a SVPSR gives a phase-space wave function closely related to the Fock-Bargmann wave function and the square magnitude of the phase-space wave function equals (apart from a constant) the Husimi distribution. Therefore, many of the results we present in this paper will contain results about the FockBargmann and Husimi representations as a special cases. Finally, we will also show that the Wigner formulation of quantum mechanics plays an important role in understanding a SVPSR based on Eq. (1).

Although from a slightly different point of view, the multitude of state-vector representations giving rise to Eq. (1) has also been considered by Harriman ${ }^{19}$ (see also an earlier paper of Torres-Vega and Frederick ${ }^{20}$ ). Harriman, however, concentrates most of his analysis on the Glauber coherent-state representation. He points out that the set of possible phase-space wave functions formed with this choice of basis only constitutes a subset of $\mathscr{L}^{2}(2)$, and that this subset can be characterized by studying certain phase-space eigenvalue equations. Our analysis generalizes these observations and provides additional insight into the origin of these eigenvalue equations. Furthermore, we describe how these eigenvalue equations can be utilized as a tool to perform wave mechanics in phase space in an unambiguous way.
The remainder of this paper is organized as follows. In Sec. II we introduce coherent states as basis vectors in a phase-space representation (PSR). We show that any coherent-state representation (CSR) gives rise to the operator mapping in Eq. (1). And, finally, by relating the CSRs to the position and momentum representations we argue-using the work of Harriman ${ }^{19}$ - that the CSRs are, in fact, the only representations that give rise to Eq. (1). We also discuss the interpretation of the square magnitude as a phase-space density and the possibilities of using this density to calculate expectation values as phase-space averages. In Sec. III we consider the possibilities and limitations of doing wave mechanics in phase space by solving a Schrödinger equation based on Eq. (1). We examine the implications of the ambiguity in the solutions to this equation and argue that this ambiguity, in certain cases, may be removed by supplying the Schrödinger equation with an additional differential equation. Furthermore, we show how the Schrödinger equation and the auxiliary equation can be turned into one equation in the Glauber coherent-state representation using the Fock-Bargmann representation. The main points are illustrated by examples. We summarize our findings in Sec. IV.

\section{THE COHERENT-STATE REPRESENTATIONS}

Following Klauder and Skagerstam ${ }^{5}$ and Perelomov ${ }^{6}$ a set of coherent states for the Heisenberg-Weyl group is defined as the set of states obtained by application of the Weyl (or displacement) operator

$$
D(q, p)=\exp \left\{\frac{i}{\hbar}(p Q-q P)\right\}, \quad(q, p) \in \mathrm{R} \times \mathrm{R}
$$

to any normalized vector $|\chi\rangle$. A set of coherent states is (over-)complete and may therefore be used as a basis in a state-vector representation of quantum mechanics. The study of representations created in this way is the subject of interest in this paper.

\section{A. Properties of the coherent-state representations}

For a fixed $|\chi\rangle$ (denoted as the fiducial vector in the following ${ }^{5}$ ) the set of coherent states provides a continuous representation of a quantum state where the expansion coefficients can be interpreted as an $\mathscr{L}^{2}(2)$ wave function in phase space. We introduce the vectors

$$
|q, p ; \chi\rangle \equiv D(q, p)|\chi\rangle, \quad(q, p) \in \mathrm{R} \times \mathrm{R},
$$

where the dependence of the fiducial vector is shown explicitly. These vectors define a basis in which the resolution of unity takes the form 5

$$
I=\int \frac{d q d p}{2 \pi \hbar}|q, p ; \chi\rangle\langle q, p ; \chi| .
$$

The vectors $|q, p ; \chi\rangle$ may therefore be used to introduce a phase-space wave function for the state $|\psi\rangle$ defined as

$$
\psi_{\chi}(q, p) \equiv\langle q, p ; \chi \mid \psi\rangle \text {. }
$$

In the PSRs defined this way, the Weyl operator $D(\eta, \xi)$ plays the role of a translation operator in phase space just 
like the operators $\exp (i \xi Q / \hbar)$ and $\exp (-i \eta P / \hbar)$ are translation operators in the momentum and position representation, respectively. Using the well-known relation $\exp A \exp B$ $=\exp (A+B+[A, B] / 2)$, which is valid when $[A, B]$ commutes with both $A$ and $B$, we get from Eq. (3)

$$
D(\eta, \xi)|q, p ; \chi\rangle=|q+\eta, p+\xi ; \chi\rangle e^{i(\xi q-\eta p) /(2 \hbar)},
$$

where the additional phase factor stems from the noncommutativity of $Q$ and $P$.

Using Eq. (4) it thus follows that the inner product in a CSR is evaluated as

$$
\langle\psi \mid \phi\rangle=\int \frac{d q d p}{2 \pi \hbar} \psi_{\chi}^{*}(q, p) \phi_{\chi}(q, p),
$$

that is, the integration measure in a CSR is given by $(2 \pi \hbar)^{-1} d q d p$. For a normalized state $|\psi\rangle$ the phase-space wave function defined in Eq. (5) is thus normalized when integrating over phase space using this measure.

At this stage it is important to emphasize that different $|\chi\rangle$ define different SVPSRs. The change from one CSR to another is given by [using Eq. (4)]

$$
\psi_{\chi^{\prime \prime}}(q, p)=\int \frac{d q^{\prime} d p^{\prime}}{2 \pi \hbar}\left\langle q, p ; \chi^{\prime \prime} \mid q^{\prime}, p^{\prime} ; \chi^{\prime}\right\rangle \psi_{\chi^{\prime}}\left(q^{\prime}, p^{\prime}\right) .
$$

Setting $\left|\chi^{\prime \prime}\right\rangle=\left|\chi^{\prime}\right\rangle$ this relation also shows that $\left\langle q, p ; \chi^{\prime} \mid q^{\prime}, p^{\prime} ; \chi^{\prime}\right\rangle$ plays the role of a $\delta$-function in the coherent state basis $\left|q, p ; \chi^{\prime}\right\rangle$. This, however, does not imply that the states $\left|q, p ; \chi^{\prime}\right\rangle$ and $\left|q^{\prime}, p^{\prime} ; \chi^{\prime}\right\rangle$ are orthogonal. ${ }^{6}$ Common to all the CSRs is that a quantum state is represented as an $\mathscr{L}^{2}(2)$ function and integrals like $(2 \pi \hbar)^{-1} \int d q d p \psi_{\chi^{\prime}}^{*}(q, p) \phi_{\chi^{\prime \prime}}(q, p)$ of course exist but they do not qualify as a quantum-mechanical inner product. In fact, one has ${ }^{5}$

$$
\int \frac{d q d p}{2 \pi \hbar} \psi_{\chi^{\prime}}^{*}(q, p) \phi_{\chi^{\prime \prime}}(q, p)=\langle\psi \mid \phi\rangle\left\langle\chi^{\prime \prime} \mid \chi^{\prime}\right\rangle
$$

From this we learn that the phase-space representatives $\psi_{\chi^{\prime}}(q, p)$ and $\psi_{\chi^{\prime \prime}}(q, p)$ of $|\psi\rangle$ cannot have the same functional form unless $\left|\chi^{\prime}\right\rangle=\left|\chi^{\prime \prime}\right\rangle$. We also learn that $\psi_{\chi^{\prime}}(q, p)$ and $\phi_{\chi^{\prime \prime}}(q, p)$ are orthogonal in $\mathscr{L}^{2}(2)$ if the states $|\psi\rangle$ and $|\phi\rangle$ are orthogonal, but we cannot conclude the reverse since the two fiducial vectors $\left|\chi^{\prime}\right\rangle$ and $\left|\chi^{\prime \prime}\right\rangle$ might be orthogonal.

Expressions for the operators $Q$ and $P$ in a coherent state representation can be found using the relations ${ }^{5,6}$

$$
\begin{aligned}
& D^{\dagger}(q, p) Q=\left(\frac{q}{2}+i \hbar \frac{\partial}{\partial p}\right) D^{\dagger}(q, p), \\
& D^{\dagger}(q, p) P=\left(\frac{p}{2}-i \hbar \frac{\partial}{\partial q}\right) D^{\dagger}(q, p) .
\end{aligned}
$$

These relations are easily proved by differentiation of the decompositions

$$
\begin{aligned}
D^{\dagger}(q, p) & =e^{i p q /(2 \hbar)} e^{i q P / \hbar} e^{-i p Q / \hbar}, \\
& =e^{-i p q /(2 \hbar)} e^{-i p Q / \hbar} e^{i q P / \hbar},
\end{aligned}
$$

with respect to $p$ and $q$, respectively. We then get for any fiducial vector $|\chi\rangle$ that

$$
\begin{aligned}
\left\langle q, p ; \chi\left|Q^{n}\right| \psi\right\rangle & =\left\langle\chi\left|D^{\dagger}(q, p) Q^{n}\right| \psi\right\rangle, \\
& =\left(\frac{q}{2}+i \hbar \frac{\partial}{\partial p}\right)^{n}\langle q, p ; \chi \mid \psi\rangle, \\
\left\langle q, p ; \chi\left|P^{n}\right| \psi\right\rangle & =\left\langle\chi\left|D^{\dagger}(q, p) P^{n}\right| \psi\right\rangle, \\
& =\left(\frac{p}{2}-i \hbar \frac{\partial}{\partial q}\right)^{n}\langle q, p ; \chi \mid \psi\rangle,
\end{aligned}
$$

which are just the forms anticipated in Refs. $12-17$ and 19. Thus for a (Taylor-expandable) operator $\Omega(Q, P)$ we have

$$
\Omega(Q, P) \mapsto \Omega\left(\frac{q}{2}+i \hbar \frac{\partial}{\partial p}, \frac{p}{2}-i \hbar \frac{\partial}{\partial q}\right) .
$$

\section{B. Relation to the position and momentum representations}

The phase-space wave function in Eq. (5) is defined without reference to any other representation and in this way the CSRs qualify as "true" state-vector representations. However, all state-vector representations are equivalent and a transition from one representation to another may be accomplished by use of the resolution of the identity of the representation which is departed from. Specifically, a transition from the CSRs to the position- or momentum-space representation is effected by

$$
\begin{aligned}
& \psi\left(q^{\prime}\right)=\left\langle q^{\prime} \mid \psi\right\rangle=\int \frac{d q d p}{2 \pi \hbar}\left\langle q^{\prime} \mid q, p ; \chi\right\rangle \psi_{\chi}(q, p), \\
& \widetilde{\psi}\left(p^{\prime}\right)=\left\langle p^{\prime} \mid \psi\right\rangle=\int \frac{d q d p}{2 \pi \hbar}\left\langle p^{\prime} \mid q, p ; \chi\right\rangle \psi_{\chi}(q, p),
\end{aligned}
$$

where the coherent-state version of the resolution of the identity has been used. These integrals may be regarded as scalar products in phase space between the phase-space wave function and the eigenstates of $Q$ and $P$, respectively, in the coherent-state basis defined by $|\chi\rangle$. Similarly, the reverse transition is accomplished by using the position and momentum version of the resolution of the identity $I=\int d q^{\prime}\left|q^{\prime}\right\rangle\left\langle q^{\prime}\right|$ and $I=\int d p^{\prime}\left|p^{\prime}\right\rangle\left\langle p^{\prime}\right|$, respectively, i.e.,

$$
\begin{aligned}
\psi_{\chi}(q, p) & \equiv\langle q, p ; \chi \mid \psi\rangle, \\
& =\int d q^{\prime}\left\langle q, p ; \chi \mid q^{\prime}\right\rangle \psi\left(q^{\prime}\right), \\
& =\int d p^{\prime}\left\langle q, p ; \chi \mid p^{\prime}\right\rangle \widetilde{\psi}\left(p^{\prime}\right) .
\end{aligned}
$$

These integrals can be regarded as scalar products in position [momentum] space between $\psi\left(q^{\prime}\right)\left[\widetilde{\psi}\left(p^{\prime}\right)\right]$ and a coherent state parametrized by $p$ and $q$ in the position [momentum] representation. Without specifying the fiducial vector we do, of course, not have explicit expressions for the transition 
elements $\left\langle q, p ; \chi \mid q^{\prime}\right\rangle$ and $\left\langle q, p ; \chi \mid p^{\prime}\right\rangle$. However, from the properties of the displacement operator we have for any $|\chi\rangle$ that

$$
\begin{aligned}
& \left\langle q, p ; \chi \mid q^{\prime}\right\rangle=\left\langle q^{\prime}-q \mid \chi\right\rangle^{*} e^{-i p\left(q^{\prime}-q / 2\right) / \hbar}, \\
& \left\langle q, p ; \chi \mid p^{\prime}\right\rangle=\left\langle p^{\prime}-p \mid \chi\right\rangle^{*} e^{i q\left(p^{\prime}-p / 2\right) / \hbar} .
\end{aligned}
$$

These are eigenstates of $Q$ and $P$ in a coherent-state basis and they are easily seen to satisfy

$$
\begin{aligned}
& \left(\frac{q}{2}+i \hbar \frac{\partial}{\partial p}\right)\left\langle q, p ; \chi \mid q^{\prime}\right\rangle=q^{\prime}\left\langle q, p ; \chi \mid q^{\prime}\right\rangle, \\
& \left(\frac{p}{2}-i \hbar \frac{\partial}{\partial q}\right)\left\langle q, p ; \chi \mid p^{\prime}\right\rangle=p^{\prime}\left\langle q, p ; \chi \mid p^{\prime}\right\rangle .
\end{aligned}
$$

Using the phase-space scalar product, we find that the phasespace eigenfunctions of $Q$ and $P$ are $\delta$-function normalized as they should be. For instance,

$$
\begin{aligned}
& \int \frac{d q d p}{2 \pi \hbar}\left\langle q^{\prime \prime} \mid q, p ; \chi\right\rangle\left\langle q, p ; \chi \mid q^{\prime}\right\rangle, \\
& =\int \frac{d q d p}{2 \pi \hbar}\left\langle\chi \mid q^{\prime}-q\right\rangle\left\langle q^{\prime \prime}-q \mid \chi\right\rangle e^{i p\left(q^{\prime \prime}-q^{\prime}\right) / \hbar}, \\
& =\delta\left(q^{\prime \prime}-q^{\prime}\right) \int d q\left\langle\chi \mid q^{\prime}-q\right\rangle\left\langle q^{\prime \prime}-q \mid \chi\right\rangle, \\
& =\delta\left(q^{\prime \prime}-q^{\prime}\right),
\end{aligned}
$$

where we have used that $\int d q\left\langle\chi \mid q^{\prime}-q\right\rangle\left\langle q^{\prime \prime}\right.$ $-\left.q|\chi\rangle\right|_{q^{\prime \prime}=q^{\prime}}=\langle\chi \mid \chi\rangle=1$. Similarly, we find that

$$
\int \frac{d q d p}{2 \pi \hbar}\left\langle p^{\prime \prime} \mid q, p ; \chi\right\rangle\left\langle q, p ; \chi \mid p^{\prime}\right\rangle=\delta\left(p^{\prime \prime}-p^{\prime}\right) .
$$

Also by direct evaluation in phase space we find

$$
\int \frac{d q d p}{2 \pi \hbar}\left\langle p^{\prime} \mid q, p ; \chi\right\rangle\left\langle q, p ; \chi \mid q^{\prime}\right\rangle=\frac{e^{-i p^{\prime} q^{\prime} / \hbar}}{\sqrt{2 \pi \hbar}}
$$

as expected. ${ }^{21}$

At this stage we can make a connection to the work of Harriman ${ }^{19}$ in order to show that the CSRs are the only representations in which the operators $Q$ and $P$ take the form in Eq. (1). Harriman assumes the existence of a phase-space representation where the position and momentum operators take the form given by Eq. (1). Based on the form of these operators, he finds the linear maps between the position- and momentum-space representations and the phase-space representation. In fact, Harriman finds infinitely many maps of this type from the position- and momentum-space representations to a phase-space representation, where the fundamental operators assume the anticipated form. This implies that there is not a single but infinitely many of such phase-space representations.
From the anticipated form of the fundamental operators in the phase-space representations and in, say, the positionspace representation, Harriman is able to derive a formal expression for the linear map between the phase-space representations and the position-space representation. A similar expression for the linear map between the phase-space representations and the momentum-space representation is obtained. Now, the interesting thing is that these formal expressions for the linear maps coincide with those for the transition elements Eq. (16) above. Since the expressions of Harriman were derived from "first principles," we conclude that the CSRs discussed above are the only ones where the position and momentum operators take the form Eq. (1).

As mentioned in the introduction, Torres-Vega and co-workers ${ }^{12-17}$ have previously introduced a phase-space state-vector representation based on the postulate that there exists a set of basis vectors $|\Gamma\rangle=|q, p\rangle$ with the closure relation $I=\int d \Gamma|\Gamma\rangle\langle\Gamma|$ where they can define the operators of $Q$ and $P$ to take the form given in Eq. (1). With the conclusion above it is clear that Torres-Vega and co-workers do not describe a single representation but rather a class of representations, namely the CSRs, and we can then identify their basis vector with any of the vectors $|q, p ; \chi\rangle$ divided by $(2 \pi \hbar)^{1 / 2}$. This identification provides useful insight into the work of Torres-Vega and co-workers. For instance, it is now evident that the infinite number of fiducial vectors is responsible for the ambiguity in the phase-space wave functions that they observe. Furthermore, one of the key points in their work was that their PSR cannot be obtained from the position- or momentum-space representations, one can only go the other way around, that is, the position- or momentumspace wave functions can be obtained from the phase-space wave function by a projection. As demonstrated above, this is certainly not true [cf. Eqs. (14) and (15)]. However, these procedures require knowledge of the fiducial vector-a concept that was not recognized in their work-and without this knowledge Eqs. (14) and (15) are useless. On the other hand, by comparing the Schrödinger equation in position, momentum, and phase space Torres-Vega and Frederick ${ }^{12}$ found that the position- and momentum-space wave functions can be obtained from the phase-space wave function by "Fourier-like" projections. These procedures must therefore be fiducial-vector independent and, in fact, we get from Eq. (15) that

$$
\begin{aligned}
& \frac{1}{2 \pi \hbar} \int d p e^{i p q /(2 \hbar)} \psi_{\chi}(q, p), \\
& =\frac{1}{2 \pi \hbar} \int d q^{\prime}\left\langle\chi \mid q^{\prime}-q\right\rangle\left\langle q^{\prime} \mid \psi\right\rangle \int d p e^{-i p\left(q^{\prime}-q\right) / \hbar}, \\
& =\int d q^{\prime}\left\langle\chi \mid q^{\prime}-q\right\rangle\left\langle q^{\prime} \mid \psi\right\rangle \delta\left(q^{\prime}-q\right), \\
& =\left.\langle\chi \mid q\rangle\right|_{q=0}\langle q \mid \psi\rangle,
\end{aligned}
$$




$$
\begin{aligned}
\frac{1}{2 \pi \hbar} & \int d q e^{-i p q /(2 \hbar)} \psi_{\chi}(q, p), \\
& =\frac{1}{2 \pi \hbar} \int d p^{\prime}\left\langle\chi \mid p^{\prime}-p\right\rangle\left\langle p^{\prime} \mid \psi\right\rangle \int d q e^{i q\left(p^{\prime}-p\right) / \hbar}, \\
& =\int d p^{\prime}\left\langle\chi \mid p^{\prime}-p\right\rangle\left\langle p^{\prime} \mid \psi\right\rangle \delta\left(p^{\prime}-p\right), \\
& =\left.\langle\chi \mid p\rangle\right|_{p=0}\langle p \mid \psi\rangle,
\end{aligned}
$$

where the fiducial vector only shows up as a constant on the right-hand side. This may, however, be zero if the wave function $\langle q \mid \chi\rangle[\langle p \mid \chi\rangle]$ vanishes at $q=0[p=0]$. This demonstrates that the information contained in the phase-space wave function about the fiducial vector is in some sense redundant.

This may be compared to the work of Wlodarz ${ }^{22}$ who demonstrates that the Wigner function may be expressed as the so-called $\star$-product of a phase-space wave function and its complex conjugate. The phase-space wave functions considered by Wlodarz may be shown to be wave functions (with rescaled arguments) in any CSR, a fact implicitly recognized by Wlodarz by identifying the time-evolution equation for the wave functions in his approach with the one put forward by Torres-Vega and Frederick. ${ }^{12}$ Hence, for computing the Wigner function the fiducial-vector information in the CSR wave function is again somewhat redundant.

In any case, in order to give the phase-space wave function a meaningful interpretation-and for some computational purposes-knowledge of fiducial vector is imperative.

\section{Interpretation of the phase-space wave function}

Like any other representation, the CSRs introduce a probability amplitude or wave function, here denoted $\psi_{\chi}(q, p)$. The "universal" interpretation of such a quantity is, of course, that its square magnitude gives the probability of the state under consideration being in a basis state of the representation specified by some labels or parameters. In the present case,

$$
\left|\psi_{\chi}(q, p)\right|^{2}=|\langle q, p ; \chi \mid \psi\rangle|^{2}
$$

gives the probability of the state $|\psi\rangle$ being in the coherent state $|q, p ; \chi\rangle$. The labels are here $q, p$, and $\chi$.

The association of a CSR with a PSR assumes that some physical significance has been attributed to two of the three labels above, namely $q$ and $p$. The same pertains to the usual position- and momentum-space representations. Moreover, in these representations the respective wave functions are associated with a probability measure directly related to the involved labels, i.e., in the position-space representation, for instance, $|\psi(q)|^{2} d q$ gives the probability of observing the position of the system between $q$ and $q+d q$. In this way, not just the label $q$ in the position-space wave function is attributed a physical significance but also the wave function itself is-namely the well-known fact that the square magnitude $|\psi(q)|^{2}$ is considered a probability density.

Such an interpretation of the position-space wave function is formally enabled by the fact that the corresponding representation diagonalizes the position operator. A similar argument holds for the momentum-space representation. Now, although considering a normalized phase-space wave function, thus giving rise to some density in phase space, a similar physical significance cannot be "tied" to a phasespace wave function in a simple manner. First of all, because in any CSR none of the fundamental operators are diagonalized (both operators are non-local) and, second, because of the so far unspecified parameter $\chi$ of the fiducial vector. So, what sort of information does a phase-space wave function offer with respect to its labels?

To answer this question, let us first briefly examine the structure of such a wave function. In the cases where the fiducial vector is a ground state of any physical harmonic oscillator the square magnitude $\left|\psi_{\chi}(q, p)\right|^{2}$ is easily seen to be the well-known Husimi function. ${ }^{23,8}$ Hence, for a general fiducial vector the density is of a "Husimi-type" construction.

As discussed by e.g., Stenholm ${ }^{24}$ and Royer, ${ }^{25}$ in their analyses of the Wigner function, a phase-space density resulting from a simultaneous measurement of position and momentum is obtained as the convolution of the Wigner function for the system under consideration with the Wigner function of a "test body", 24 or probe state with negated arguments, rather than the system Wigner function itself. This convolution, given by

$$
2 \pi \hbar \int d q^{\prime} d p^{\prime} W_{\chi}\left(q^{\prime}-q, p^{\prime}-p\right) W_{\psi}\left(q^{\prime}, p^{\prime}\right)
$$

where $W_{\chi}(q, p)$ is the Wigner function for the probe state and $W_{\psi}(q, p)$ is the Wigner function for the system in question, constitutes a "fuzzy" phase-space density subject to the uncertainty principle. ${ }^{25}$ Equation (24) is seen to equal the square magnitude Eq. (23) when the fiducial vector $|\chi\rangle$ represents the probe state.

Thus, $\left|\psi_{\chi}(q, p)\right|^{2}$ is a "fuzzy" phase-space density and, according to Royer, ${ }^{25}$ this density gives for each pair of labels $(q, p)$ the relative probability that the system is localized in a "fuzzy" neighbourhood of the centre of the displaced probe state (fiducial vector). ${ }^{26}$ Two things should be noted about this interpretation. First, we have in the general case that

$$
\langle\chi|Q| \chi\rangle=q_{\chi}, \quad\langle\chi|P| \chi\rangle=p_{\chi},
$$

and only when the fiducial vector is physically centred, i.e., only when $q_{\chi}=p_{\chi}=0$ the label pair $(q, p)$ denotes the centre of the displaced probe state. In general, the centre is $\left(q+q_{\chi}, p+p_{\chi}\right)$. Therefore, the label point $(q, p)$ in a plot of a general density $\left|\psi_{\chi}(q, p)\right|^{2}$ is a "statement" about the physical point $\left(q+q_{\chi}, p+p_{\chi}\right)$. Second, the meaning of a " 'fuzzy neighbourhood" needs to be specified. If the fiducial vector is a ground state for some harmonic oscillator the density $\left|\psi_{\chi}(q, p)\right|^{2}$ represents the most precise description of 
the system since the probe state, in this case, is a minimum uncertainty state (MUS) ${ }^{24}$ - the uncertainties in position and momentum of the fiducial vector determine, in a sense, how sharply our description is resolved. Hence, it seems reasonable to associate the "fuzzy neighbourhood" with the uncertainty region around the centre of the displaced probe state. In other words, if the fiducial vector is a physically centred MUS the square magnitude $\left|\psi_{\chi}(q, p)\right|^{2}$ gives the probability of finding the system inside a phase-space volume $\Delta q \Delta p=h$ around the point $(q, p)$.

While such an interpretation is intuitively very appealing for a localized fiducial vector as the MUS, and obviously in accordance with the uncertainty principle, it is perhaps less satisfactory for other more "diffuse" fiducial vectors. Consider, for instance, the case where the fiducial vector is an odd- $n$ eigenstate for any physical harmonic oscillator. The position- and momentum-space densities attribute vanishing probability of the fiducial vector being at position zero or having zero momentum. Nevertheless, the expected values are zero. In such cases the above characterization of the density $\left|\psi_{\chi}(q, p)\right|^{2}$ seems to be a poor one; based only on the first two moments of the fundamental operators in the fiducial vector.

Therefore, the mathematically precise answer to the question posed earlier in this section is: The density $\left|\psi_{\chi}(q, p)\right|^{2}$ gives the probability of having the position- and momentum-space densities $\left|\left\langle q^{\prime}-q \mid \chi\right\rangle\right|^{2}$ and $\left|\left\langle p^{\prime}-p \mid \chi\right\rangle\right|^{2}$, respectively, i.e., the position- and momentum-space densities of the fiducial vector.

\section{Expectation values using the phase-space density}

According to Eq. (4), the expectation value of the operator $\Omega(Q, P)$ in the state $|\psi\rangle$ can be calculated in any CSR as is usually done in other state-vector representations, viz.

$$
\begin{aligned}
\langle\psi|\Omega(Q, P)| \psi\rangle= & \int \frac{d q d p}{2 \pi \hbar} \psi_{\chi}^{*}(q, p) \Omega\left(\frac{q}{2}+i \hbar \frac{\partial}{\partial p}, \frac{p}{2}\right. \\
& \left.-i \hbar \frac{\partial}{\partial q}\right) \psi_{\chi}(q, p) .
\end{aligned}
$$

However, let us consider the possibility of using the density itself for calculating expectation values as integrals over phase space. $^{27}$ To this end we again turn to the Wigner phase-space formulation in which the expectation value of the operator $\Omega(Q, P)$ in the state $|\psi\rangle$ is expressed by the phase-space integral

$$
\langle\psi|\Omega(Q, P)| \psi\rangle=\int d q d p \Omega_{W}(q, p) W_{\psi}(q, p) .
$$

The function $\Omega_{W}(q, p)$ is the Weyl-transform of the operator $\Omega(Q, P) .^{7}$ If we require that the expectation value of $\Omega(Q, P)$ should be expressed in a similar fashion when using $\left|\psi_{\chi}(q, p)\right|^{2}$ as the density it is expected that the function representing the observable in this case must depend on the fiducial vector. In fact, one readily finds using Eq. (24) that expectation value of the operator $\Omega(Q, P)$ in the state $|\psi\rangle$ may be expressed as

$$
\langle\psi|\Omega(Q, P)| \psi\rangle=\int \frac{d q d p}{2 \pi \hbar} \Omega_{\chi}(q, p)\left|\psi_{\chi}(q, p)\right|^{2},
$$

where the function $\Omega_{\chi}(q, p)$ is given implicitly by

$$
\Omega_{W}(q, p)=\int d q^{\prime} d p^{\prime} W_{\chi}\left(q-q^{\prime}, p-p^{\prime}\right) \Omega_{\chi}\left(q^{\prime}, p^{\prime}\right) .
$$

Unfortunately, though, the class of operators $\Omega(Q, P)$ for which the functions $\Omega_{\chi}(q, p)$ exist in general is highly restricted and, even when they exist, the integral, Eq. (29), may be difficult to invert as noted by several authors in somewhat different frameworks (see e.g., the discussions in Refs. 7 and 8 of the existence and evaluation of $\Omega_{\chi}(q, p)$ for some special choices of fiducial vectors).

However, for " tors, that is, for $Q^{n}$ and $P^{n}$ which under the Weyl-transform simply become $q^{n}$ and $p^{n}$, respectively, the integrals are easily inverted for any fiducial vector. Upon performing the substitution $q-q^{\prime} \mapsto q^{\prime}$, Eq. (29) becomes in the case of the position operator $Q$

$$
Q_{W}^{n} \equiv q^{n}=\int d q^{\prime} d p^{\prime} Q_{\chi}^{n}\left(q-q^{\prime}, p-p^{\prime}\right) W_{\chi}\left(q^{\prime}, p^{\prime}\right),
$$

where $Q_{\chi}^{n}$ is the function for $Q^{n}$ in the $\chi$-CSR. A reasonable ansatz for this function would be

$$
Q_{\chi}^{n}\left(q-q^{\prime}, p-p^{\prime}\right)=\sum_{k=0}^{n} a_{k}\left(q-q^{\prime}\right)^{k} .
$$

Substituting back into Eq. (30) and using that $\int d p W_{\psi}(q, p)=|\psi(q)|^{2}$ for any state $|\psi\rangle$ we arrive at

$$
q^{n}=\sum_{k=0}^{n} a_{k}\left\langle(q-Q)^{k}\right\rangle_{\chi}
$$

where \langle\rangle$_{\chi}$ denotes the expected value with respect to the fiducial vector $|\chi\rangle$. Clearly, $a_{n}$ must equal unity, whence we are left with a system of $n$ linear homogeneous algebraic equations in the coefficients $a_{0}, \ldots, a_{n-1}$.

The procedure is completely equivalent for "pure", powers of the momentum operator $P$, and for the first and second powers we arrive at the mappings

$$
\begin{array}{ll}
Q \mapsto q+q_{\chi}, & Q^{2} \mapsto\left(q+q_{\chi}\right)^{2}-(\Delta q)_{\chi}^{2}, \\
P \mapsto q+q_{\chi}, & P^{2} \mapsto\left(p+p_{\chi}\right)^{2}-(\Delta p)_{\chi}^{2},
\end{array}
$$

where the right-hand sides give the functions to be used in Eq. (28). Here, $x_{\chi} \equiv\langle X\rangle_{\chi}$ is the expected value and $(\Delta x)_{\chi}^{2} \equiv\left\langle X^{2}\right\rangle_{\chi}-\langle X\rangle_{\chi}^{2}$ the variance, both with respect to the fiducial vector.

A few remarks about these results are appropriate. For a physically centred MUS fiducial vector Eq. (33) coincides with results of Harriman and Casida. ${ }^{8}$ Hence, Eq. (32) and its momentum equivalent is a generalization of these authors' results to the use of an arbitrary fiducial vector. Second, again for a physically centred MUS fiducial vector the function $\Omega_{\chi}\left(q^{\prime}, p^{\prime}\right)$ is recovered as the Glauber-Sudarshan 
$P$-symbol or anti-normally ordered symbol, used in quantum optics, ${ }^{6,7,28}$ of the operator $\Omega$. Therefore, for an arbitrary fiducial vector $\Omega_{\chi}\left(q^{\prime}, p^{\prime}\right)$ represents a generalized $P$-symbol in accordance with the interpretation of the CSRdensity $\left|\psi_{\chi}(q, p)\right|^{2}$ as being a generalized Husimi representation or, to use the quantum optics terminology, a generalized $Q$-representation. This, in turn, reflects the dual nature of the $Q$ - and $P$-representations, also in the generalized sense (see also Dahl ${ }^{29,30}$ ). In view of this fact it should be noted that use of the term "Husimi representation" for the function $\Omega_{\chi}\left(q^{\prime}, p^{\prime}\right)$ by Harriman and Casida ${ }^{8}$ is somewhat misguiding, giving the impression that the Husimi representation is a self-dual PSR.

Finally, it should be noted that the presented relations Eqs. (24) and (29) between the generalized Husimi, WeylWigner, and Glauber-Sudarshan symbols provide an interesting insight into the connections between these symbols and representations - again for the standard versions as well as the generalized ones. It is well known ${ }^{31}$ that in the standard cases the Weyl-Wigner symbol may be obtained from the Glauber-Sudarshan symbol by a Gaussian smearing or convolution and that the Husimi symbol (apart from a multiplicative factor) is obtained from the Weyl-Wigner symbol by a smearing with same Gaussian. Furthermore, we know from the Husimi symbol that the widths of the smearing Gaussian reflect the uncertainties in position and momentum of the MUS fiducial vector. Since the Husimi function for a MUS too is a Gaussian so are the Wigner and Glauber-Sudarshan functions. Hence, the smearing Gaussian in the standard formulations could be any of the three symbols for the MUS fiducial vector. However, from Eqs. (24) and (29) we conclude that, in the general cases, the smearing function is the Wigner function for the fiducial vector. So the Wigner function of the fiducial vector provides the link between the three mentioned symbols of any operator, and in this respect the Weyl-Wigner representation may be considered the more fundamental one.

\section{WAVE MECHANICS IN PHASE SPACE}

In this section we consider the possibility and implications of doing wave mechanics in phase space, that is, solving the equation of motion in a CSR. For a Hamiltonian of the type $H(Q, P)=P^{2} /(2 m)+V(Q)$ the equation of motion governing the dynamics of a phase-space wave function is the phase-space Schrödinger equation,

$$
\begin{aligned}
i \hbar \frac{\partial}{\partial t} \psi_{\chi}(q, p)= & {\left[\frac{1}{2 m}\left(\frac{p}{2}-i \hbar \frac{\partial}{\partial q}\right)^{2}\right.} \\
& \left.+V\left(\frac{q}{2}+i \hbar \frac{\partial}{\partial p}\right)\right] \psi_{\chi}(q, p) .
\end{aligned}
$$

The equation is the same for any fiducial vector and the solution of this equation will therefore yield infinite number of phase-space wave functions-corresponding to the infinite number of CSRs-describing the same physical state. The ambiguity of the fiducial vector clearly arises because the operator mapping Eq. (1) only depends on the form of the Weyl operator Eq. (2) and not the fiducial vector itself.

Two questions now arise:

(i) How do we know to which fiducial vector a particular solution of the Schrödinger equation belongs? and

(ii) Is this important for practical purposes?

The second question can be answered immediately when "practical purposes" is explicitly defined. The questions asked in quantum mechanics are ultimately questions about observables or dynamical variables. By "practical purposes" we, accordingly, mean the use of wave functions for calculating expectation values and transition matrix elements.

Hence, for two solutions, $\psi_{\chi}$ and $\phi_{\chi^{\prime}}$, of Eq. (34) and presumably different fiducial vectors, $|\chi\rangle$ and $\left|\chi^{\prime}\right\rangle$, the "matrix elements" of an operator $\Omega(Q, P)$ may be computed by application of Eq. (9) as

$$
\begin{aligned}
& \int \frac{d q d p}{2 \pi \hbar} \psi_{\chi}^{*}(q, p) \Omega\left(\frac{q}{2}+i \hbar \frac{\partial}{\partial p}, \frac{p}{2}-i \hbar \frac{\partial}{\partial q}\right) \phi_{\chi^{\prime}}(q, p) \\
& =\langle\psi|\Omega(Q, P)| \phi\rangle\left\langle\chi^{\prime} \mid \chi\right\rangle .
\end{aligned}
$$

Therefore, in order to calculate the matrix element using phase-space wave functions one has to be sure that the two wave functions belong to the same CSR. In the calculation of expectation values this is trivially fulfilled since the same wave function is used twice, so to speak. Hence, if only expectation values are needed the answer to question (ii) above is "no"; otherwise "yes."

This brings us back to the first question and the immediate answer to that is: "We don't." An additional criterion is needed in order to assure that the two chosen wave functions belong to the same CSR. In order to be a useful criterion it must force a condition on the fiducial part of the wave function only and not on the state vector part.

Such a criterion is provided by requiring that the fiducial vector be an eigenstate of a non-degenerate Taylor expandable operator, say $A_{\chi}(Q, P)$, such that $|\chi\rangle$ is uniquely determined from the eigenvalue equation

$$
A_{\chi}(Q, P)|\chi\rangle=a_{\chi}|\chi\rangle,
$$

once the eigenvalue $a_{\chi}$ is specified. This equation is clearly formulated without reference to any state vector. Upon employing the properties of the displacement operator, Eq. (36) may be turned into an equation in the CSR based on the fiducial vector $|\chi\rangle$. Explicitly we have

$$
\begin{aligned}
& {\left[D(q, p) A_{\chi}(Q, P) D^{\dagger}(q, p)\right]|q, p ; \chi\rangle} \\
& \quad=A_{\chi}(Q-q, P-p)|q, p ; \chi\rangle=a_{\chi}|q, p ; \chi\rangle,
\end{aligned}
$$

which results in the phase-space eigenvalue equation for any state $|\psi\rangle$

$$
A_{\chi}^{*}\left(-\frac{q}{2}+i \hbar \frac{\partial}{\partial p},-\frac{p}{2}-i \hbar \frac{\partial}{\partial q}\right) \psi_{\chi}(q, p)=a_{\chi}^{*} \psi_{\chi}(q, p) .
$$

This equation constitutes the additional requirement. Hence, for those fiducial vectors which are eigenvectors for some operator $A_{\chi}(Q, P)$, we may classify the solutions of the 
phase-space Schrödinger equation. One way to do so is to "prune" the set of solutions to the Schrödinger equation with the auxiliary equation for a specific $a_{\chi}^{*}$. Equation (38) is a generalization of the two phase-space eigenvalue equations set up by Harriman ${ }^{19}$ in the Glauber coherent-state representation (where the fiducial vector is a physically centred MUS). The two equations set up by Harriman corresponds to $A_{\chi}$ being the annihilator with $a_{\chi}=0$ and $A_{\chi}$ being the Hamiltonian for harmonic oscillator with $a_{\chi}=1 / 2$.

Of course, the Glauber coherent-state representation is a very important one but as discussed briefly by Klauder and Skagerstam 5 other choices of fiducial vector are not only of academic interest. The above procedure has been employed in the study of the spectrum of the quartic oscillator (see Ref. 5 and Refs. 65-71 therein). The approach taken in these investigations is that of choosing the fiducial vector to be one of the unknown eigenstates of the quartic oscillator. Thus, a natural choice of $A_{\chi}(Q, P)$ would be the Hamiltonian itself, rendering the auxiliary equation Eq. (38) the conjugate of the stationary Schrödinger equation. The combining of these two equations leads to differential equations for the eigenfunctions where the fiducial vectors are well-known, in the sense that they too are eigenfunctions of the Hamiltonian and therefore may be labeled. In this way the Schrödinger equation and the auxiliary equation are solved simultaneously.

In conclusion, to do wave mechanics in phase space in an unambiguous way, i.e., for a given fiducial vector according to the above described scheme, we have to solve two phase-space equations - either simultaneously or in a successive manner. This should be contrasted to wave mechanics in the usual Schrödinger representation. Here, a single equation is sufficient.

\section{A. The Fock-Bargmann approach}

The question is if it is possible to device a scheme in phase space where a single equation suffices to solve the quantum problem and to fix the representation. Clearly, such a scheme cannot be based on the operator mapping Eq. (1); a mapping in unique correspondence with a fiducial vector must be chosen. One such mapping has been employed by Skodje, Rohrs, and VanBuskirk ${ }^{32}$ for a MUS fiducial vector. However, in the following we shall devise another scheme based on the famous Fock-Bargmann representation mentioned in the Introduction.

The Fock-Bargmann representation (FBR) constitutes a state-vector representation in the complex plane based on a mapping of a pair of boson operators $\left(a, a^{\dagger}\right)$. In this representation, quantum mechanics can be performed unambiguously due to the fact that it diagonalizes the creation operator $a^{\dagger}$. Hence, wave mechanics in phase space can be performed in an unambiguous manner, similar to the standard Schrödinger representation, via the FBR if this can be linked to the CSRs discussed so far.

Such a link is provided by the representation in the coherent states of Glauber. ${ }^{33}$ Being originally formulated in terms of the boson operators, this representation is intimately connected with the FBR. At the same time the Glauber representation is closely related to the $(q, p)$-parametrized CSR with a physically centred MUS as fiducial vector, whence the FBR provides a means for doing wave mechanics in phase space based on the class of $(q, p)$-parametrized CSRs with a physically centred MUS as fiducial vector.

At this point it should be mentioned that it is possible to set up a generalized procedure since (i) the only restriction on the relation between the boson operators and the "physical" operators $Q$ and $P$ is that $\left[a, a^{\dagger}\right]=1$ and since (ii) a Fock-Bargmann-type representation can be associated with any Glauber-type representation based on a fiducial vector that is an eigenstate of $a .^{6}$ This enlarges the class of $(q, p)$-parametrized CSRs, that may be handled via a FBR, to including those based on a not physically centred fiducial vector. However, the purpose of introducing the FBR in the present context is to enable a simple and convenient scheme for doing wave mechanics in phase space and with reference to the discussion in the preceding section, $(q, p)$ -parametrized CSRs with a physically centred MUS as fiducial vector constitute the physically most appealing statevector PSRs. Accordingly, we shall restrict ourselves to the standard procedure.

Thus, the boson operators are expressed in terms of $Q$ and $P$ in the standard form

$$
\begin{aligned}
& a=\frac{1}{\sqrt{2 \hbar}}\left(\lambda Q+i \lambda^{-1} P\right), \\
& a^{\dagger}=\frac{1}{\sqrt{2 \hbar}}\left(\lambda Q-i \lambda^{-1} P\right),
\end{aligned}
$$

and with the Glauber coherent states given by

$$
|\alpha\rangle=D(\alpha)|0\rangle, \quad D(\alpha)=\exp \left(\alpha a^{\dagger}-\alpha^{*} a\right),
$$

where $a|0\rangle=0$ and where $\alpha=\left(\lambda q+i \lambda^{-1} p\right) / \sqrt{2 \hbar}$, the relationship between wave functions in the Glauber and FockBargmann representations is

$$
\psi\left(\alpha, \alpha^{*}\right) \equiv\langle\alpha \mid \psi\rangle=\exp \left(-|\alpha|^{2} / 2\right) \psi_{\mathrm{FB}}(z), z \equiv \alpha^{*} .
$$

Finally, the boson operators are in the FBR mapped according to

$$
a \mapsto \frac{\partial}{\partial z}, \quad a^{\dagger} \mapsto z \quad \text { standard FBR. }
$$

Based on the above considerations we, therefore, propose the following scheme for doing wave mechanics in phase space in an unambiguous Schrödinger-like manner, that is, wave mechanics in a CSR based on a MUS fiducial vector. The fiducial vector is completely fixed by a parameter $\lambda$ which, however, may be chosen at will.

(i) Given a Hamiltonian $H(Q, P)$ we can choose a map between the fundamental operators $(Q, P)$ and the boson operators $\left(a, a^{\dagger}\right)$ according to Eq. (39) by specifying the parameter $\lambda \in R$. In this way the Hamiltonian may be re-expressed as

$$
H(Q, P) \mapsto \mathscr{H}\left(a, a^{\dagger}\right)
$$

(ii) By this and by using the standard Fock-Bargmann operator mapping Eq. (42) we have chosen a specific 
FBR, that corresponds to a physically centred $(q, p)$-parametrized CSR. In other words, the choice of $\lambda$ fixes the fiducial vector and hence the representation. In this FBR the Schrödinger equation is set up according to

$$
\begin{aligned}
i \hbar \frac{\partial}{\partial t}|\psi\rangle= & \mathscr{H}\left(a, a^{\dagger}\right)|\psi\rangle \\
& \mapsto i \hbar \frac{\partial}{\partial t} \psi_{\mathrm{FB}}(z)=\mathscr{H}(\partial / \partial z, z) \psi_{\mathrm{FB}}(z) .
\end{aligned}
$$

Here $z=\left(\lambda q-i \lambda^{-1} p\right) / \sqrt{2 \hbar}$. It is emphasized that the solutions to this equation are unambiguously tied to the FBR representation automatically selected by the choice of mapping between $(Q, P)$ and $\left(a, a^{\dagger}\right)$ when the standard FockBargmann operator mapping Eq. (42) is used.

(iii) Therefore, a FBR wave function $\psi_{\mathrm{FB}}(z)$ is uniquely associated with a wave function $\psi_{\chi}(q, p)$ in a physically centred $(q, p)$-parametrized CSR with the explicit relation being

$$
\psi_{\chi}(q, p)=\exp \left(-|z|^{2} / 2\right) \psi_{\mathrm{FB}}(z), \quad|\chi\rangle=|0\rangle .
$$

These steps show that a complete set of wave functions in the class of CSRs with a MUS fiducial vector may be obtained unambiguously by solving the Schrödinger equation in the standard FBR.

We conclude this subsection by noting that the unique correspondence between an operator mapping and a fiducial vector is equivalent to an implicit fulfillment of the auxiliary equation Eq. (38). The power of the Fock-Bargmann representation is that this condition is fulfilled by construction.

\section{B. Examples}

The three standard examples to consider are the free particle, the linear potential and the harmonic oscillator. Since the free-particle eigenfunctions equal the eigenfunctions of the momentum operator, these are already given as the lower expression in Eq. (16). Torres-Vega and co-workers have previously considered several examples of analytic solutions to the phase-space Schrödinger equation; coherent states and eigenstates of the harmonic oscillator ${ }^{12,16}$ and, most recently, eigenfunctions of the linear potential. ${ }^{17}$ In the following we analyze the linear and quadratic potential in terms of CSRs in order to provide more insight into the previous results. It turns out that all the wave functions of Torres-Vega and co-workers are given in CSRs based on MUSs.

\section{Eigenstates of a linear potential}

Torres-Vega et al. ${ }^{17}$ have recently considered a set of solutions to the stationary phase-space Schrödinger equation,

$\left[\frac{1}{2 m}\left(\frac{p}{2}-i \hbar \frac{\partial}{\partial q}\right)^{2}+K\left(\frac{q}{2}+i \hbar \frac{\partial}{\partial p}\right)\right] \psi_{E}(q, p)=E \psi_{E}(q, p)$.

The reason to take this problem up again is to analyze the findings in Ref. 17 in terms of CSRs and to illustrate one of the drawbacks of working directly with the phase-space Schrödinger equation arising from the lack of "control" of the fiducial vector.
Let us briefly summarize the procedure of determining phase-space eigenfunctions of the linear potential used by Torres-Vega et al. They introduce the dimensionless coordinates $x=\beta(q-2 E / K)$ and $y=p /(\beta \hbar)$ where $\beta=\left(2 m K / \hbar^{2}\right)^{1 / 3}$ in order to re-write the stationary phasespace Schrödinger equation as

$$
\left[\left(\frac{y}{2}-i \frac{\partial}{\partial x}\right)^{2}+\left(\frac{x}{2}+i \frac{\partial}{\partial y}\right)\right] \phi(x, y)=0,
$$

where $\phi(x, y)=\psi_{E}(q(x, E ; \beta), p(y ; \beta))$. A set of solutions to this equation is then given in the form

$$
\phi(x, y)=N e^{-i x y / 2} \int d t e^{i t^{3} / 3-\alpha(t-y)^{2} / 2+i t x},
$$

where $\alpha$ is an arbitrary non-negative parameter and $N$ is a normalization constant. This gives rise to the phase-space eigenfunctions

$$
\begin{aligned}
\psi_{E}(q, p)= & N e^{-i p(q-2 E / K) /(2 \hbar)} \\
& \times \int d t e^{i t^{3} / 3-\alpha[t-p /(\beta \hbar)]^{2} / 2+i t \beta(q-2 E / K)} .
\end{aligned}
$$

Now, let us see which fiducial vector that leads to this phase-space wave function. Starting from, for instance, the position-space eigenfunctions, ${ }^{34}$

$$
\left\langle q \mid \psi_{E}\right\rangle=\frac{\beta}{2 \pi \sqrt{K}} \int d t e^{i t^{3} / 3+i t \beta(q-E / K)},
$$

it is straightforward to show that with the fiducial vector being a MUS centred at $q=-E / K$ and $p=0$, which in position space takes the form

$$
\langle q \mid \chi\rangle=\left(\frac{\lambda^{2}}{\pi \hbar}\right)^{1 / 4} \exp \left\{-\frac{\lambda^{2}}{2 \hbar}(q+E / K)^{2}\right\}
$$

one obtains the phase-space eigenfunctions of Torres-Vega, Zúñiga-Segundo, and Morales-Guzmán using the identification $\alpha=\hbar(\beta / \lambda)^{2}$. The parameter $\alpha$ therefore depends on both the physical system and the properties of the fiducial vector. For a given set of $(m, K)$ and $\hbar$, the value of $\alpha$ is thus fixes $\lambda$. However, fixation of $\lambda$ only determines the uncertainty properties of the fiducial vector; its centering is determined by the energy. This implies that even for a fixed $\alpha$ the phase-space energy eigenfunctions of Torres-Vega, ZúñigaSegundo, and Morales-Guzmán do not belong to the same representation. In fact, they all belong to different representations! This shows that since the phase-space Schrödinger does not contain any information about the fiducial vector one has given up the "control" of the representation by working directly with this equation.

To ensure that the different eigenfunctions belong to the same representation one has to find the relationship between the different eigenstates in abstract Hilbert space and then choose a single representation for all of them. In abstract Hilbert space, the Schrödinger equation is

$$
\left[\frac{P^{2}}{2 m}+K(Q-E / K)\right]\left|\psi_{E}\right\rangle=0 \text {. }
$$


Using the well-known relations ${ }^{33}$

$$
\begin{aligned}
& D^{\dagger}(\eta, \xi) Q D(\eta, \xi)=Q+\eta, \\
& D^{\dagger}(\eta, \xi) P D(\eta, \xi)=P+\xi,
\end{aligned}
$$

this equation can be re-written as

$$
\left[\frac{P^{2}}{2 m}+K Q\right] D(-E / K, 0)\left|\psi_{E}\right\rangle=0 .
$$

Therefore, in abstract Hilbert space, $\left|\psi_{E}\right\rangle=D(E / K, 0)\left|\psi_{0}\right\rangle$. Since $\langle q| D(\eta, 0)=\langle q-\eta|$, the position-space eigenfunctions with eigenenergy $E$ are simply obtained from the $E=0$ eigenfunction by a displacement of the position coordinate, as seen in Eq. (50). In any CSR the relation is, using Eq. (6),

$$
\psi_{E}(q, p)=\psi_{0}(q-E / K, p) e^{-i p E /(2 \hbar K)},
$$

which is a displacement in position and a momentumdependent change of phase. Equation (55) also shows that square magnitude of the eigenstates in any CSR obey the "classical" relation between energy and translation along the position direction in phase space. The wave functions in Eq. (49) do not satisfy Eq. (55) illustrating that they cannot belong to the same phase-space representation.

However, we readily deduce from above analysis the complete set of phase-space eigenfunctions with the fiducial vector being, for instance, the ground state of a physical oscillator $|0\rangle$ with $q^{\prime}=p^{\prime}=0$. These are given by

$$
\psi_{E}(q, p)=\phi[\beta(q-E / K), p /(\beta \hbar)] e^{-i p E /(2 \hbar K)}
$$

or

$$
\begin{aligned}
\psi_{E}(q, p)= & N e^{-i p q /(2 \hbar)} \\
& \times \int d t e^{i t^{3} / 3-\alpha[t-p /(\beta \hbar)]^{2} / 2+i t \beta(q-E / K)} .
\end{aligned}
$$

With this choice of the fiducial vector, the square magnitude of these wave functions equals the Husimi function. In Ref. 17 the square magnitude of $\phi(x, y)$ is studied as $\alpha$ is varied. Having related $\alpha$ to the shape of the fiducial vector through $\lambda$, the findings in Ref. 17 are in accordance with the interpretations in Sec. II C, namely that high resolution is obtained in the position direction for small $\alpha$ (where the uncertainty of the fiducial vector in the position direction is also small), whereas high resolution is obtained in the momentum direction for large $\alpha$. It also only in this limit that the square magnitude of the eigenstates (being independent of $q$ and $p$ ) are strictly stationary states of the classical equations of motion.

Finally, let us demonstrate how $\psi_{0}(q, p)$ with the fiducial vector being a physically centred MUS can be found by solving the stationary FBR Schrödinger equation. Following the procedure of the preceding subsection we find that the FBR wave function satisfy

$$
\begin{aligned}
& \left\{\frac{d^{2}}{d z^{2}}-2\left[2(\alpha / 2)^{3 / 2}+z\right] \frac{d}{d z}+\left[z^{2}-4(\alpha / 2)^{3 / 2} z-1\right]\right\} \\
& \quad \times \psi_{0}(z)=0,
\end{aligned}
$$

where again $\alpha=\hbar(\beta / \lambda)^{2}$. A physically centred MUS is fully specified through $\lambda$ and, therefore, for a given set of the physical parameters $\hbar$ and $\beta$ the choice of the value for $\alpha$ is a choice of CSR.

With the transformations ${ }^{35}$

$$
\begin{aligned}
& \psi_{0}(z)=e^{z^{2} / 2+2(\alpha / 2)^{3 / 2} z} \phi(w), \\
& w=\sqrt{2 \alpha} z+(\alpha / 2)^{2},
\end{aligned}
$$

the equation for $\phi(w)$ becomes the usual differential equation for the Airy function,

$$
\left(\frac{d^{2}}{d w^{2}}-w\right) \phi(w)=0 .
$$

Using Eq. (45) we can, therefore, write the eigenstate of a linear potential with $E=0$ in the CSRs, with fiducial vectors being physically centred MUSs, in terms of the usual Airy function as

$$
\psi_{0}(q, p)=N e^{-\left(|z|^{2}-z^{2}\right) / 2+2(\alpha / 2)^{3 / 2} z} \times \operatorname{Ai}\left[\sqrt{2 \alpha} z+(\alpha / 2)^{2}\right],
$$

where, according to the remarks following Eq. (58),

$$
z=\frac{\beta}{\sqrt{2 \alpha}} q-i \frac{\sqrt{\alpha / 2}}{\hbar \beta} p .
$$

Therefore, the relation between $z$ and $(q, p)$ is determined once $\hbar, \alpha$, and $\beta$ are specified. Thus, the complete set of eigenfunctions can be obtained using Eq. (55). That the phase-space wave functions in these representations can be expressed in terms of well-known functions is not evident from previous results.

\section{The harmonic oscillator}

The harmonic oscillator has been treated by Torres-Vega and co-workers in Refs. 12 and 15 paying special attention to the correspondence between the quantum phase-space dynamics and classical dynamics. In the following we address this point from the point of view of CSRs using a MUS as fiducial vector. In order to obtain the same notation as used by Torres-Vega and co-workers, we redefine the parameter characterizing the shape of the fiducial vector according to $\lambda^{2}=(1+2 \alpha) /(1-2 \alpha)$. For simplicity, let the Hamiltonian be written in a rescaled form, $H(Q, P)=\left(P^{2}+Q^{2}\right) / 2$.

With the fiducial vector being a physically centred MUS, the time-dependent phase-space wave function of a Glauber coherent state may be found directly from Ref. 36 as

$$
\begin{aligned}
\psi_{t}(q, p)= & \left(1-4 \alpha^{2}\right)^{1 / 4} \exp \left[-\frac{1+2 \alpha}{4 \hbar}\left(q-q_{t}\right)^{2}\right. \\
& -\frac{1-2 \alpha}{4 \hbar}\left(p-p_{t}\right)^{2}+\frac{i}{2 \hbar}\left(q p_{t}-p q_{t}\right) \\
& \left.-\frac{i \alpha}{\hbar}\left(q-q_{t}\right)\left(p-p_{t}\right)-\frac{i}{2} t\right],
\end{aligned}
$$

provided that the parameters $q_{t}$ and $p_{t}$ satisfy the classical equations of motion $\dot{q}_{t}=p_{t}$ and $\dot{p}_{t}=-q_{t}$, that is, 
(a)

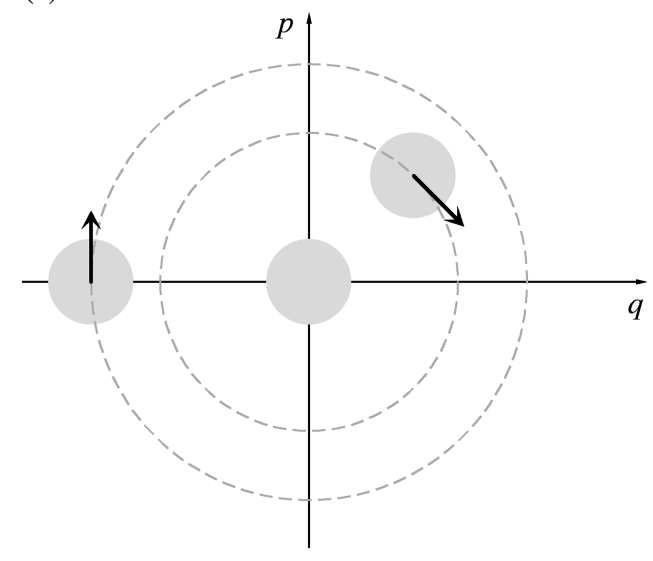

(b)

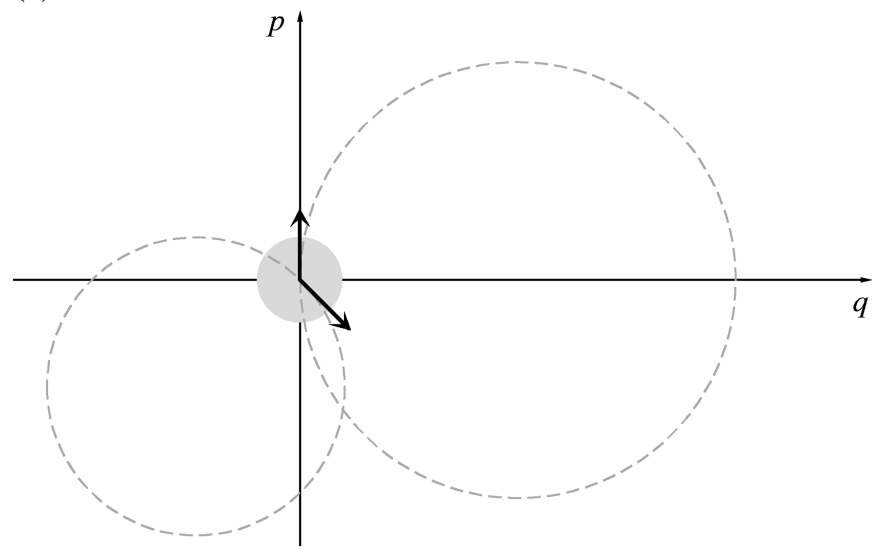

FIG. 1. Schematic figure of the "classical" motion of three different coherent states of a harmonic oscillator in different CSRs. In (a) the coherent states are depicted in the same CSR based on a physically centred MUS fiducial vector. In (b) the coherent states are shown in CSRs with fiducial vectors coinciding with the coherent states themselves at time zero. See text for details. The arrows indicate the direction of the phase velocity as the states travel along the dashed circles.

$$
\begin{aligned}
& q_{t}=q_{0} \cos t+p_{0} \sin t, \\
& p_{t}=p_{0} \cos t-q_{0} \sin t .
\end{aligned}
$$

Equation (63), which is easily seen to satisfy the phase-space Schrödinger equation, is the one found in Ref. 15. The phase-space density, as given by the square magnitude of Eq. (63), is

$$
\begin{aligned}
\left|\psi_{t}(q, p)\right|^{2}= & \left(1-4 \alpha^{2}\right)^{1 / 2} \exp \left[-\frac{1+2 \alpha}{2 \hbar}\left(q-q_{t}\right)^{2}\right. \\
& \left.-\frac{1-2 \alpha}{2 \hbar}\left(p-p_{t}\right)^{2}\right] .
\end{aligned}
$$

The density is thus a Gaussian centred on the classical orbit. The axes are aligned with the coordinate axes at all times, with their lengths controlled by $\alpha$. Since the classical dynamics in a rescaled oscillator describes rigid rotations about the origin, the density in Eq. (65) only obeys classical evolution for $\alpha=0(\lambda=1)$. For this value of $\alpha$ the fiducial vector is the ground state of the rescaled harmonic oscillator.

The physical properties of the wave functions, Eq. (63), are, of course, independent of $\alpha$. For any $\alpha$ we find, e.g., that $\left\langle\psi_{t}|Q| \psi_{t}\right\rangle=q_{t},\left\langle\psi_{t}|P| \psi_{t}\right\rangle=p_{t}$ and $(\Delta Q)_{t}^{2}=(\Delta P)_{t}^{2}=\hbar / 4$ Thus, all the wave functions in Eq. (63) are coherent states, and not squeezed states as stated in Ref. 15, despite the elliptic contours in phase space of their square magnitudes. Their different shapes do not reflect different uncertainties in the fundamental operators but different choices of the fiducial vector!

To illustrate how the choice of a not physically centred fiducial vector might be confusing, let us consider as fiducial vector a MUS centred at the arbitrary point $\left(q^{\prime}, p^{\prime}\right)$. The shape density of the coherent states are still Gaussian but it will be displaced by $-q^{\prime}\left[-p^{\prime}\right]$ in the position [momentum] direction in comparison with the density in Eq. (65). This implies that the density will evolve as though it is subject to a harmonic potential with the vertex of the parabola centred at $\left(-q^{\prime},-p^{\prime}\right)$. For instance, in the CSR where the fiducial vector is centred at $\left(q_{0}, p_{0}\right)$ the phase-space wave function representing a coherent state at time $t=0$ becomes

$$
\begin{aligned}
\psi_{0}(q, p)= & \left(1-4 \alpha^{2}\right)^{1 / 4} \exp \left[-\frac{1+2 \alpha}{4 \hbar} q^{2}-\frac{1-2 \alpha}{4 \hbar} p^{2}\right. \\
& \left.-\frac{i}{2 \hbar}\left(q p_{0}-p q_{0}\right)-\frac{i \alpha}{\hbar} q p\right],
\end{aligned}
$$

which gives rise to a Gaussian density centred at the origin. But, the initial phase velocity is still given by $\mathbf{v}_{0}=\left(p_{0},-q_{0}\right)$ and the density will move along the circle centred at $\left(-q_{0},-p_{0}\right)$. In Fig. 1(a) we have shown, in the standard CSR, contours of three different coherent states at time $t=0$ along with the direction of their initial phasevelocity vectors, and a sketch of the phase-space orbits they will follow as time evolves. In Fig. 1(b) the same coherent states are shown in the CSRs defined above, that is, the state vector and the fiducial vector being the same.

These examples show how important the knowledge of the fiducial vector is when one wishes to interpret the phasespace density. Especially, we see that of the set of fiducial vectors considered here-minimum uncertainty states-only a single one will result in classical dynamics for the phasespace density of a coherent-state in a harmonic potential, namely the fiducial vector being equal to the ground state of the considered harmonic oscillator. As may be deduced from Eq. (33) in Ref. 36, this is true for any PSR eigenstate of the harmonic potential; a fact also observed, albeit not explained, by Torres-Vega and Morales-Guzmán. ${ }^{15}$

In fact, the square magnitude of any phase-space wave function will evolve classically in an at most quadratic potential if the fiducial vector is chosen to be a physically centred MUS with the "right" shape. From the general (fiducial-vector independent) equation of motion for the density $^{12}$ this is not easily recognized, but with a specific choice of fiducial vector the knowledge of the fiducial vector 
can be build into the equation of motion, and with the fiducial vector being a physically centred MUS (where the density is a Husimi function) the equation of motion for the density can be written as the classical Liouville equation plus correction terms. ${ }^{10,32}$

For a potential of the kind

$$
V(Q)=k_{0}+k_{1} Q+\frac{1}{2} k_{2} Q^{2}
$$

the square magnitude of any phase-space wave function in this class of CSRs satisfies

$$
\begin{aligned}
\frac{\partial}{\partial t}\left|\psi_{t}(q, p)\right|^{2}= & \left(-\frac{p}{m} \frac{\partial}{\partial q}+\frac{\partial V_{c}}{\partial q} \frac{\partial}{\partial p}\right)\left|\psi_{t}(q, p)\right|^{2} \\
& +\hbar\left(\frac{1}{2 \lambda^{2}} \frac{\partial^{2} V_{c}}{\partial q^{2}}-\frac{\lambda^{2}}{2 m}\right) \frac{\partial^{2}}{\partial q \partial p}\left|\psi_{t}(q, p)\right|^{2},
\end{aligned}
$$

where $V_{c}=V(q)$. Independently of $\hbar$, this equation reduces to the Liouville equation for $\lambda=\left(m k_{2}\right)^{1 / 4}$. This implies that the phase-space density for a free particle and a particle in a linear potential will only undergo classical evolution in the limit $\lambda \rightarrow 0$ (as seen above for the stationary states of the linear potential), and for a harmonic oscillator the phasespace density evolves classically if the fiducial vector is the ground state of the oscillator. In virtue of Ehrenfest's Theorem and Eq. (33), the centre of any density based on a physically centred fiducial vector will evolve classically in the potential Eq. (67), but only for a specific choice of such a fiducial vector the density as a whole will behave classically. ${ }^{37}$ It is, furthermore, evident from the equation of motion of the Husimi function ${ }^{10,32}$ that if the potential has non-vanishing derivatives of higher order than two no choice of MUS as fiducial vector will result in classical evolution of the phase-space density.

If the MUS is not physically centred the dynamics in the situations described above will still be "classical-like" in the sense that the density will move along trajectories that are solutions to Hamilton's equation, however with a the Hamiltonian, cf. Eq. (33),

$$
H(q, p)=\frac{1}{2 m}\left(p+p_{\chi}\right)^{2}+V\left(q+q_{\chi}\right) .
$$

Thus for a finite $\hbar$ the CSR will only behave classicallike for certain potentials and with special choices of the fiducial vector. Recently, Klauder ${ }^{38}$ has examined the dynamics of the CSR density for $\hbar$ going to zero. ${ }^{39}$ He demonstrated that if the fiducial vector has a vanishing dispersion in this limit the dynamics of any state in any potential will be classical-like in the above sense as $\hbar$ goes to zero.

\section{DISCUSSION AND CONCLUDING REMARKS}

In the coherent-state representations (CSRs), as formulated by Klauder and Skagerstam, ${ }^{5}$ wave functions are $\mathscr{L}^{2}(2)$ functions of the parameters $q$ and $p$ and the fundamental operators $Q$ and $P$ are mapped according to Eq. (1), irrespectively of the fiducial vectors. From these fundamen- tal properties we have demonstrated that the state-vector representation formulated by Torres-Vega and co-workers, ${ }^{12-17}$ and subsequently analyzed by Harriman, ${ }^{19}$ comprises the class of all coherent-state representations. In other words, any quantum state is represented by infinitely many $\mathscr{b}^{2}(2)$ functions, each one belonging to a specific fiducial vector.

This observation has spawned several questions: How should such phase-space wave functions be interpreted, how are they related to other representations, how can wave mechanics be done in phase space, and how do phase-space wave functions evolve in time? Each of these questions have been addressed with particular emphasis on the role played by the fiducial vector.

Clearly, since the square magnitudes of wave functions in the standard CSR, i.e., the one where the fiducial vector is a minimum-uncertainty state (MUS) with vanishing expected values of position and momentum, are Husimi functions, densities in any CSR must be Husimi-like functions. As for the standard Husimi function the interpretation of any CSR density as a probability density in phase space is troublesome. However, following Royer, ${ }^{25}$ a CSR density may be considered a "fuzzy" density in phase space, at each point $(q, p)$ giving the relative probability of the system being situated in a "fuzzy" neighbourhood of the centre of $D(q, p)|\chi\rangle$. Precisely how this "fuzzy" neighbourhood is shaped and where it is positioned depends on the properties of the fiducial vector. Such an interpretation suggests that information about the system may be extracted from images in phase space. However, extreme care should be exercised. For instance, only for physically centred fiducial vectors the point $(q, p)$ represents the actual physical point in phase space. Moreover, the notion of a "fuzzy" neighbourhood seems to be descriptive only for localized fiducial vectors, such as MUSs. For choices of other more "diffuse" fiducial vectors very little information about the system is provided by the phase-space images. Accordingly, we infer that images of CSR densities are descriptive only in the cases where the fiducial vector is localized. Still knowledge about the fiducial vector is crucial as was demonstrated by the examples in Sec. III.B..

Being "true"' state-vector representations, the CSRs admit the possibility of solving a quantum problem directly in phase space. However, because of the fiducial-vector independence of the mapping of the fundamental operators, e.g., the stationary Schrödinger equation for a given problem assumes the same form in any CSR. Accordingly, any solution to this equation could belong to any CSR; to which we do not know. As we have discussed, this is not a problem if one merely wishes to compute expectation values whereas explicit knowledge about the fiducial vector is required for calculating transition matrix elements. To resolve this ambiguity we have suggested two approaches. Either one may augment the Schrödinger equation with an auxiliary equation that fixes the fiducial vector. Or one may invoke the FockBargmann representation. While the former approach necessitates two equations to be solved it is applicable for any fiducial vector that admits the set-up of the auxiliary equation. Conversely, the Fock-Bargmann representation can 
only be put in one-to-one correspondence with CSRs based on MUS fiducial vector, but only solution of a single equation is required. In either scheme knowledge about the fiducial vector is provided. The "pitfalls" encountered upon just solving the phase-space Schrödinger equation and the resolution of the ambiguity obtained by employing the FockBargmann approach were illustrated in Sec. III B in the case of a linear potential.

As the standard Husimi function may be used for computing expectation values as integrals over phase space, so may any CSR density. This requires a mapping of the operator in question into a function of the parameters $q$ and $p$. Unlike the one for the CSRs, this mapping is fiducial-vector dependent and in the cases the operator being "pure" powers of either of the fundamental operators a procedure for obtaining these phase-space functions was given. The first two powers of both $Q$ and $P$ were stated explicitly and reduced to the previously reported results for the usual Husimi representation. ${ }^{8}$ Apart from merely devicing the operator map the procedure also provided insight into the nature of these generalized Husimi representations and their connections to the Weyl-Wigner representation. For instance, the dual nature of the Husimi representation, also in the generalized case, was demonstrated explicitly by the form of the operator map which, in fact, defines a generalized GlauberSudarshan $P$-representation. Moreover, it was shown that the function "linking" the Husimi, Weyl-Wigner, and GlauberSudarshan representations together is the Wigner function, and not any of the other symbols, of the fiducial vector chosen. Hence, another instance giving rise to considering the Wigner representation the more fundamental densityoperator phase-space representation.

In conclusion, the present analysis revealed that the phase-space representation of Torres-Vega and co-workers ${ }^{12-17}$ coincide with the class of all CSRs, as formulated by Klauder and Skagerstam, ${ }^{5}$ that these representations are related to the position and momentum representations in standard Diracian manner, and that the fiducial vector plays a prominent and crucial role in the CSRs that must be recognized when images of CSR densities are to be interpreted and when one desires to do wave mechanics in phase space.

\section{ACKNOWLEDGMENTS}

The authors would like to thank Dr. J. J. Włodarz and Professor J. P. Dahl for careful reading of the manuscript and enlightening comments. This research was supported by The Danish Natural Science Foundation.

\footnotetext{
${ }^{1}$ V. Fock, Z. Phys. 49, 339 (1928).

${ }^{2}$ V. Bargmann, Commun. Pure Appl. Math 14, 187 (1961).

${ }^{3}$ E. Wigner, Phys. Rev. 40, 749 (1932).

${ }^{4}$ K. Husimi, Proc. Phys. Math. Soc. Jpn. 22, 264 (1940).

${ }^{5}$ J. R. Klauder and B.-S. Skagerstam, Coherent States (World Scientific, Singapore, 1985).
}

${ }^{6}$ A. Perelomov, Generalized Coherent States and Their Applications: Texts and Monographs in Physics (Springer Verlag, Berlin, 1986).

${ }^{7}$ M. Hillery, R. F. O'Connell, M. O. Scully, and E. P. Wigner, Phys. Rep. 106, 121 (1984).

${ }^{8}$ J. E. Harriman and M. E. Casida, Int. J. Quantum Chem. 45, 263 (1993).

${ }^{9}$ M. V. Berry, Philos. Trans. R. Soc. London, Ser. A 287, 237 (1977).

${ }^{10}$ K. Takahashi, J. Phys. Soc. Jpn. 55, 762 (1986).

${ }^{11}$ A. Voros, Phys. Rev. A 40, 6814 (1989).

${ }^{12}$ Go. Torres-Vega and J. H. Frederick, J. Chem. Phys. 98, 3103 (1993).

${ }^{13}$ Go. Torres-Vega, J. Chem. Phys. 98, 7040 (1993).

${ }^{14}$ Go. Torres-Vega, J. Chem. Phys. 99, 1824 (1993).

${ }^{15}$ Go. Torres-Vega, in Third International Workshop on Squeezed States and Uncertainty Relations, edited by D. Han, Y. S. Kim, N. M. Rubin, Y. Shih, and W. W. Zachary (NASA, Maryland, 1994), pp. 275-280.

${ }^{16}$ Go. Torres-Vega and J. D. Morales-Guzmán, J. Chem. Phys. 101, 5847 (1994).

${ }^{17}$ Go. Torres-Vega, A. Zúniga-Segundo, and J. D. Morales-Guzmán, Phys. Rev. A 53, 3792 (1996).

${ }^{18}$ R. J. Glauber, Phys. Rev. 130, 2529 (1963).

${ }^{19}$ J. E. Harriman, J. Chem. Phys. 100, 3651 (1994).

${ }^{20}$ Go. Torres-Vega and J. H. Frederick, J. Chem. Phys. 93, 8862 (1990).

${ }^{21}$ P. A. M. Dirac, The Principles of Quantum Mechanics, 4th ed. (Oxford University Press, Oxford, 1958).

${ }^{22}$ J. J. Wlodarz, J. Chem. Phys. 100, 7476 (1994).

${ }^{23}$ J. E. Harriman, J. Chem. Phys. 88, 6399 (1988).

${ }^{24}$ S. Stenholm, Eur. J. Phys. 1, 244 (1980).

${ }^{25}$ A. Royer, Phys. Rev. Lett. 55, 2745 (1985).

${ }^{26}$ For a discussion of simultaneous measurements of non-commuting observables in terms of the precise mathematical concepts "fuzzy sets" and "fuzzy measures," see e.g., E. Prugoveǒki, Found. Phys. 3, 3 (1973) and E. B. Davies and J. T. Lewis, Commun. Math. Phys. 17, 239 (1970).

${ }^{27}$ This should be compared with, for instance, the position-space representation where the square magnitude of the wave function can be used to calculate the expectation value of any operator that is a function of the position operator. This is due to the fact that any such operator is multiplicative in the position-space representation. Since neither the position nor the momentum operator is multiplicative in a CSR it is not obvious whether the square magnitude of the phase-space wave function can be used in a similar way.

${ }^{28} \mathrm{P}$. Meystre and M. Sargent III, Elements of Quantum Optics, 2nd ed. (Springer-Verlag, Berlin, 1991).

${ }^{29}$ J. P. Dahl, in Classical and Quantum Systems: Foundations and Symmetries, Proceedings of the II International Wigner Symposium, edited by $\mathrm{H}$. D. Doebner, W. Scherer, and F. Schroeck, Jr. (World Scientific, Singapore, 1993), pp. 420-423.

${ }^{30}$ J. P. Dahl, in Conceptual Trends in Quantum Chemistry, edited by E. S. Kryachko and J. L. Calais (Kluwer Academic Publishers, Amsterdam, 1994), pp. 199-226.

${ }^{31}$ K. E. Cahill and R. J. Glauber, Phys. Rev. 177, 1882 (1969).

${ }^{32}$ R. T. Skodje, H. W. Rohrs, and J. VanBuskirk, Phys. Rev. A 40, 2894 (1989).

${ }^{33}$ R. J. Glauber, Phys. Rev. 131, 2766 (1963).

${ }^{34}$ L. D. Landau and E. M. Lifshitz, Quantum Mechanics, Vol. 3 of Course of Theoretical Physics, 3rd ed. (Pergamon Press Ltd., Oxford, 1977).

${ }^{35}$ D. Zwillinger, Handbook of Differential Equations (Academic, San Diego, 1989).

${ }^{36}$ K. B. Møller, T. G. Jørgensen, and J. P. Dahl, Phys. Rev. A 54, 5378 (1996).

${ }^{37}$ This should be contrasted with the Wigner density which always behaves classically in the potential Eq. (67).

${ }^{38}$ J. R. Klauder, in Workshop on Harmonic Oscillators, edited by D. Han, Y. S. Kim, and W. Zachary (NASA, Maryland, 1993), pp. 19-28.

${ }^{39}$ The analysis of Klauder only involves the explicit $\hbar$ dependence in the equations of motion and is therefore appropriate if the $\hbar$ dependence of the initial phase-space density is ignored. See also Ref. 32. 